\title{
La FMCH - pour une meilleure politique de santé
}

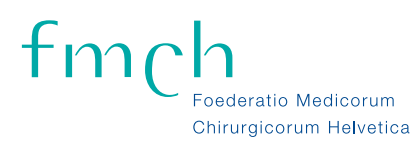

Markus Trutmann

* SSAR, SSC, SSCCVT, SSCM, SSCMF SSCP, SSCPRE, SSCR, SSDV, SSGO, SSMI, SSNC, SSO, SSO, SSORL, SSU

Correspondances: Prof. Urban Laffer Président FMCH Centre hospitalier Bienne CH-2500 Bienne

urban.laffer@fmch.ch

Dr Markus Trutmann Secrétaire général FMCH

Sonneggstrasse 84

CH-8006 Zurich

Tél. 0788360910

markus.trutmann@fmch.ch
La FMCH (Foederatio Medicorum Chirurgicorum Helvetica) est l'association faîtière de toutes les sociétés de discipline dont les membres pratiquent la chirurgie ou la médecine invasive. La FMCH fut créée le 18 septembre 2004. Elle compte aujourd'hui 16 sociétés de discipline* avec en tout plus de 5000 médecins. La FMCH est, comme la FMH, une association de droit privé et dispose de structures d'association classiques: Assemblée plénière, Comité directeur et Secrétariat général. Avec un budget annuel d'environ 1 million de francs (FMH 20 millions), la FMCH s'est dotée de moyens respectables.

\section{A quoi sert la FMCH?}

La FMCH est issue de la FMS et de l'Union des sociétés chirurgicales suisses. Elle représente les intérêts politiques des chirurgiens et des médecins pratiquant la médecine invasive. Le terme «invasif» est à prendre au sens large et inclut par exemple les anesthésistes et les médecins intensivistes. A un niveau plus général, la FMCH représente les intérêts des médecins travaillant en milieu hospitalier ou comme médecins agréés. Ces intérêts ne peuvent pas être pris en compte par la FMH de manière suffisante. Du point de vue historique, la FMH est une union de sociétés cantonales et représente surtout les intérêts des médecins de premier recours. Subissant des contraintes économiques de plus en plus lourdes, le milieu hospitalier lui aussi doit se rendre à l'évidence que ses valeurs médicales et éthiques sont menacées. Pour préserver et défendre ces valeurs, les médecins directement concernés sont décidés à s'engager eux-mêmes dans une action politique commune. La raison d'être de la FMCH est de rassembler toutes ces énergies et d'organiser une action politique efficace.

\section{La FMCH ne provoque-t-elle pas un morcellement du corps médical?}

Le rassemblement d'intérêts homogènes au sein de la FMCH évite à la FMH une épreuve de déchirement permanente. La survie de la FMH dépend directement de la réussite de la réforme des structures en cours. Or, le bon fonctionnement de la FMCH comme celui d'autres associations faîtières est la condition sine qua non de cette réussite. En face de la politique et de l'administration, la FMCH peut présenter des problèmes qui la concernent et proposer des solutions de manière plus appropriée, plus crédible et plus convaincante que la FMH. Pour toutes ces rai- sons, la FMCH ne provoque pas un morcellement, mais au contraire un renforcement du corps médical.

\section{Que fait la FMCH?}

La FMCH s'engage dans beaucoup de domaines, mais en priorité dans les trois domaines suivants:

- SwissDRG: Les membres de la FMCH sont, au contraire des médecins de premier recours et des psychiatres, directement touchés par l'introduction de SwissDRG, le nouveau système de rémunération concernant exclusivement les soins hospitaliers de cas somatiques aigus. Ainsi, la FMCH s'engage en première ligne pour prendre part activement à l'introduction de SwissDRG. De premiers succès ont pu être enregistrés l'année dernière dans le cadre de la procédure de demandes («Antragsverfahren»): toutes les propositions de la $\mathrm{FMCH}$ furent acceptées.

- Assurance-qualité: Selon la FMCH, la définition de critères de qualité est une prérogative inaliénable du corps médical. Elle refuse catégoriquement l'utilisation abusive de critères de qualité, par exemple à des fins politiques. Actuellement, la FMCH examine la possibilité de collaborer avec d'autres organisations dans le domaine de l'assurance-qualité. A cet égard, les sociétés de discipline de la FMCH se distinguent par un atout exceptionnel: elles disposent de données valables.

- Formation médicale postgrade: La FMCH s'engage pour le maintien d'une formation médicale postgrade à haut niveau tel qu'elle existe encore en Suisse. La formation médicale postgrade est menacée sur plusieurs fronts: étatisation (motion Heim), sousfinancement (SwissDRG), abaissement au niveau européen. La FMCH affronte ces dangers par des propositions aussi déterminées que constructives.

\section{Interessé?}

Si les activités de la FMCH vous intéressent ou si vous souhaitez adhérer avec votre société à la FMCH, le président, M. le Prof. Urban Laffer, Centre hospitalier de Bienne, et le secrétaire général, M. le Dr Markus Trutmann, sont très volontiers à votre disposition pour plus d'informations ou un échange d'idées. Vous êtes également invités à rendre visite à notre site web www.fmch.ch. 